Clinical paper

\title{
Hypothermia outcome prediction after extracorporeal life support for hypothermic cardiac arrest patients: The HOPE score ${ }^{\text {it }}$
}

\author{
Mathieu Pasquier ${ }^{\mathrm{a}, *}$, Olivier Hugli ${ }^{\mathrm{a}}$, Peter Paal ${ }^{\mathrm{b}}$, Tomasz Darocha ${ }^{\mathrm{c}}$, Marc Blancher ${ }^{\mathrm{d}}$, Paul Husby ${ }^{\mathrm{e}}$, \\ Tom Silfvast ${ }^{\mathrm{f}}$, Pierre-Nicolas Carron ${ }^{\mathrm{a}}$, Valentin Rousson ${ }^{\mathrm{g}}$

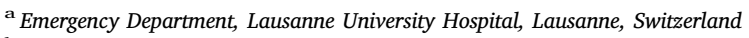 \\ ${ }^{\mathrm{b}}$ Department of Anesthesiology and Intensive Care Medicine, Hospitaller Brothers Hospital, Paracelsus Medical University, Strubergasse 21, 5020 Salzburg, Austria \\ ${ }^{\mathrm{c}}$ Severe Accidental Hypothermia Center, Department of Anaesthesiology and Intensive Care, Medical University of Silesia, 055, Poniatowskiego 15, Katowice, Poland \\ ' SAMU 38, Pôle Urgences - Médecine Aiguë, CHU de Grenoble, BP 217, 38043 Grenoble Cedex 09, France \\ e Department of Anesthesia and Intensive Care, Haukeland University Hospital, 5021 Bergen, Norway \\ ${ }^{\mathrm{f}}$ Department of Anaesthesiology, Intensive Care and Pain Medicine, University of Helsinki and Helsinki University Hospital, PO Box 340 , 00029 Helsinki, Finland \\ ${ }^{\mathrm{g}}$ Institute of Social and Preventive Medicine, Lausanne University Hospital, route de la Corniche 10, 1010 Lausanne, Switzerland
}

\section{A R T I C L E I N F O}

\section{Keywords:}

Cardiac arrest

ECMO

ECPR

Hypothermia

Accidental

Potassium

Resuscitation

Triage

\begin{abstract}
A B S T R A C T
Aims: Currently, the decision to initiate extracorporeal life support for patients who suffer cardiac arrest due to accidental hypothermia is essentially based on serum potassium level. Our goal was to build a prediction score in order to determine the probability of survival following rewarming of hypothermic arrested patients based on several covariates available at admission.

Methods: We included consecutive hypothermic arrested patients who underwent rewarming with extracorporeal life support. The sample comprised 237 patients identified through the literature from 18 studies, and 49 additional patients obtained from hospital data collection. We considered nine potential predictors of survival: age; sex; core temperature; serum potassium level; mechanism of hypothermia; cardiac rhythm at admission; witnessed cardiac arrest, rewarming method and cardiopulmonary resuscitation duration prior to the initiation of extracorporeal life support. The primary outcome parameter was survival to hospital discharge.

Results: Overall, 106 of the 286 included patients survived (37\%; 95\% CI: 32-43\%), most (84\%) with a good neurological outcome. The final score included the following variables: age, sex, core temperature at admission, serum potassium level, mechanism of cooling, and cardiopulmonary resuscitation duration. The corresponding area under the receiver operating characteristic curve was 0.895 (95\% CI: $0.859-0.931$ ) compared to 0.774 (95\% CI: $0.720-0.828$ ) when based on serum potassium level alone.

Conclusions: In this large retrospective study we found that our score was superior to dichotomous triage based on serum potassium level in assessing which hypothermic patients in cardiac arrest would benefit from extracorporeal life support. External validation of our findings is required.
\end{abstract}

\section{Introduction}

Accidental hypothermic cardiac arrest (CA) is often caused by homelessness or substance abuse, mountaineering accidents, or suicide attempts. In the United States alone, accidental hypothermia causes almost 1500 deaths annually [1] and rates of hypothermia-related deaths have been increasing [2]. However, patients who are successfully reanimated with extracorporeal life support (ECLS) rewarming often have an excellent neurological outcome [3]. ECLS is, however, clinically invasive and resource intensive, and the critical therapeutic decision to initiate rewarming of a CA patient undergoing cardiopulmonary resuscitation (CPR) often has to be made rapidly based on a very limited amount of information and ancillary examination.

This decision has been based on serum potassium levels since landmark articles on the management of accidental hypothermia demonstrated nearly three decades ago that a high serum potassium value was associated with poor outcomes [4,5]. However, the authors suggested at that time that multiple independent variables, thus far

\footnotetext{
A Spanish translated version of the abstract of this article appears as Appendix in the final online version at https://doi.org/10.1016/j.resuscitation.2018.02.026.

* Corresponding author at: Emergency Service, University Hospital Centre, BH 09, CHUV, 1011 Lausanne, Switzerland.

E-mail addresses: Mathieu.Pasquier@chuv.ch (M. Pasquier), olivier.hugli@chuv.ch (O. Hugli), peter.paal@icloud.com (P. Paal), tomekdarocha@wp.pl (T. Darocha), MBlancher@chu-grenoble.fr (M. Blancher), Paul.Husby@uib.no (P. Husby), Tom.Silfvast@hus.fi (T. Silfvast), pierre-nicolas.carron@chuv.ch (P.-N. Carron),

valentin.rousson@chuv.ch (V. Rousson).
} 
unaccounted for, probably also affected outcome. Although their conclusions regarding the practical implications of their findings were nuanced, current guidelines for CA patients experiencing accidental hypothermia or avalanche burial to date, suggest that a low serum potassium may indicate a potentially favorable prognosis [6-8] for which ECLS is indicated, while a markedly elevated level ( $>12 \mathrm{mmol} /$ $\mathrm{L}$ for accidental hypothermia and $>8 \mathrm{mmol} / \mathrm{L}$ for hypothermia due to avalanche burial) is considered an indicator of brain and heart hypoxia, secondary cell lysis, and a poor outcome $[1,4,6,9,10]$.

However, the evidence supporting serum potassium-based triage is weak, and is based mainly on case reports and series and expert opinion. By analogy with out-of-hospital cardiac arrest or other survival prediction scores [11], we postulated that the outcome and success of ECLS rewarming would depend on several additional factors [5]: for example, the mechanism and speed of induction of hypothermia and CA [12], the type of cardiac arrhythmia [12], whether the CA was witnessed [13], core temperature, and the rewarming method used [14].

Our goal was to identify and analyze the predictors of survival following ECLS rewarming among hypothermic CA patients and to develop a score in order to better predict the probability of survival of a given CA patient with accidental hypothermia based on easily available covariates.

\section{Methods}

Patients were identified and selected through a systematic literature review, and unpublished hospital data were added. We included consecutive primarily hypothermic CA victims who underwent rewarming with ECLS. To minimize the risk of inclusion bias, only consecutive ECLS patients from retrospective cohort studies or hospital data in one determined time frame were a priori included. Published cases in convenience samples or single case reports were excluded, as they were considered at risk of bias. We also excluded patients who were not in CA when ECLS was started and those for whom no individual data were available.

\section{Literature search}

We used a mixed literature research strategy. Firstly, the patients from one recent systematic review were assessed for eligibility [12]. Secondly, we updated this research with the same methodology by exploring Pubmed, Embase, and Cochrane from January 1st 2013 to December 31st 2016 using the following keywords: "Extracorporeal Membrane Oxygenation OR ECMO OR Extra-Corporeal Assisted Rewarming OR Cardiopulmonary Bypass AND Hypothermia". We also searched Pubmed for cases series using the following keywords: "Extracorporeal Membrane Oxygenation OR Cardiopulmonary Bypass AND hypothermia". We limited the research to case reports from January 1st 1987 to December 31st 2016. The last search was performed on January 5th 2017. Finally; references of retrieved papers were searched for additional patients; as were all the authors' personal libraries.

One author (MP) performed the literature search and built the database. Authors were asked for provision of individual or missing data as required, as well as for the provision of data from additional eligible unpublished patients (for details see eTable 1). Duplicate patients were excluded.

Published studies were independently and blindly assessed for potential bias by two of the authors (MP, TD). Possible disagreements were given to a third author (VR) to solve. Data from a random sample of $5 \%(n=14)$ of all included patients were randomly selected and blindly checked by one author (TD) to estimate the quality of data transcription of the main variables $(n=14)$, representing 168 single data points [15]. This resulted in $0 \%$ of discordant data between the two authors.

\section{Hospital data collection}

The data collection was approved by the institutional review board $\left(\mathrm{N}^{\circ}\right.$ 2016-01267) and have therefore been performed in accordance with the ethical standards laid down in the 1964 Declaration of Helsinki and its later amendments. Beside the addition of eligible unpublished patients provided by the authors of the retrieved studies, retrospective data from two additional hospitals were added (University Hospital, Lausanne 2000-2016 and Hospital of Sion 2004-2016, both in Switzerland).

The following data were collected for each patient: age; sex; mechanism of hypothermia; core temperature at admission (if not available, the out-of-hospital temperature was used); serum potassium level at admission; whether CA had been witnessed by rescuers or not; duration of CPR (defined from initiation of external CPR until start of ECLS); modality of ECLS rewarming (cardiopulmonary bypass (CPB) or extracorporeal membrane oxygenation (ECMO)); and CA initial rhythm at hospital admission (i.e. asystole, ventricular fibrillation or pulseless electrical activity (PEA)). In the case of CA developing after admission, the CA rhythm when starting ECLS was registered. The mechanisms for hypothermia were further classified as non-asphyxia-related (e.g. immersion, outdoor or indoor exposure to cold) or asphyxia-related (i.e., submersion, avalanche with burial of the head under the snow) [9].

All variables mentioned above were considered as potential predictors in our model. The primary outcome parameter was survival to hospital discharge [16]. A secondary outcome was the neurological outcome at hospital discharge assessed by the Cerebral Performance Category (CPC) [16]. A CPC of 1 or 2 was considered as a "favorable neurological outcome" $[17,18]$.

We termed the study design mixed, because we performed a literature search at first; and then, a data analysis complying as closely as possible with the PRISMA and TRIPOD guidelines (http://www.prismastatement.org and https://www.tripod-statement.org); and finally, we also added unpublished patients. This study was registered in the PROSPERO registry (CRD42016052882).

\section{Statistical analysis}

Univariate associations between predictors and survival were assessed using chi-square tests to compare proportions for the categorical predictors, and using a Mann-Whitney test, together with logistic regression including a quadratic term for the continuous predictors. $\mathrm{P}$ values $<0.05$ were considered as statistically significant.

In order to build our score, we started by considering a multiple logistic regression model to predict survival, which included the nine potential predictors (Additional file 2: Statistics). Since our goal was to identify the best model for prediction, we used the Akaike Information Criterion (AIC) as a model selection criterion [19]. All $p$-values for the predictors included in the best model according to AIC should be $<0.157$ [20]. Thus, at each step of a backward elimination procedure, the least significant predictor was removed and the procedure stopped when all the $p$-values associated with the remaining predictors in the model were $<0.157$ [20]. Five of the nine predictors had a few missing values, which were imputed using a MICE algorithm (Multivariate Imputation by Chained Equations) implemented in the "MICE" package (version 2.25) [21] from the $\mathrm{R}$ statistical software program [22]; this generated $\mathrm{m}=100$ imputed data sets after 50 iterations of the algorithm, where the eight remaining predictors and our response variable (survival) were all used circularly to impute the missing data for a given predictor. Therefore, each of our multiple logistic regression models were run 100 times (with the 100 different imputed data sets) while the results were aggregated using Rubin's rules [23]. Bootstrap method was used for internal validation (for details see eStatistics) [24]. 


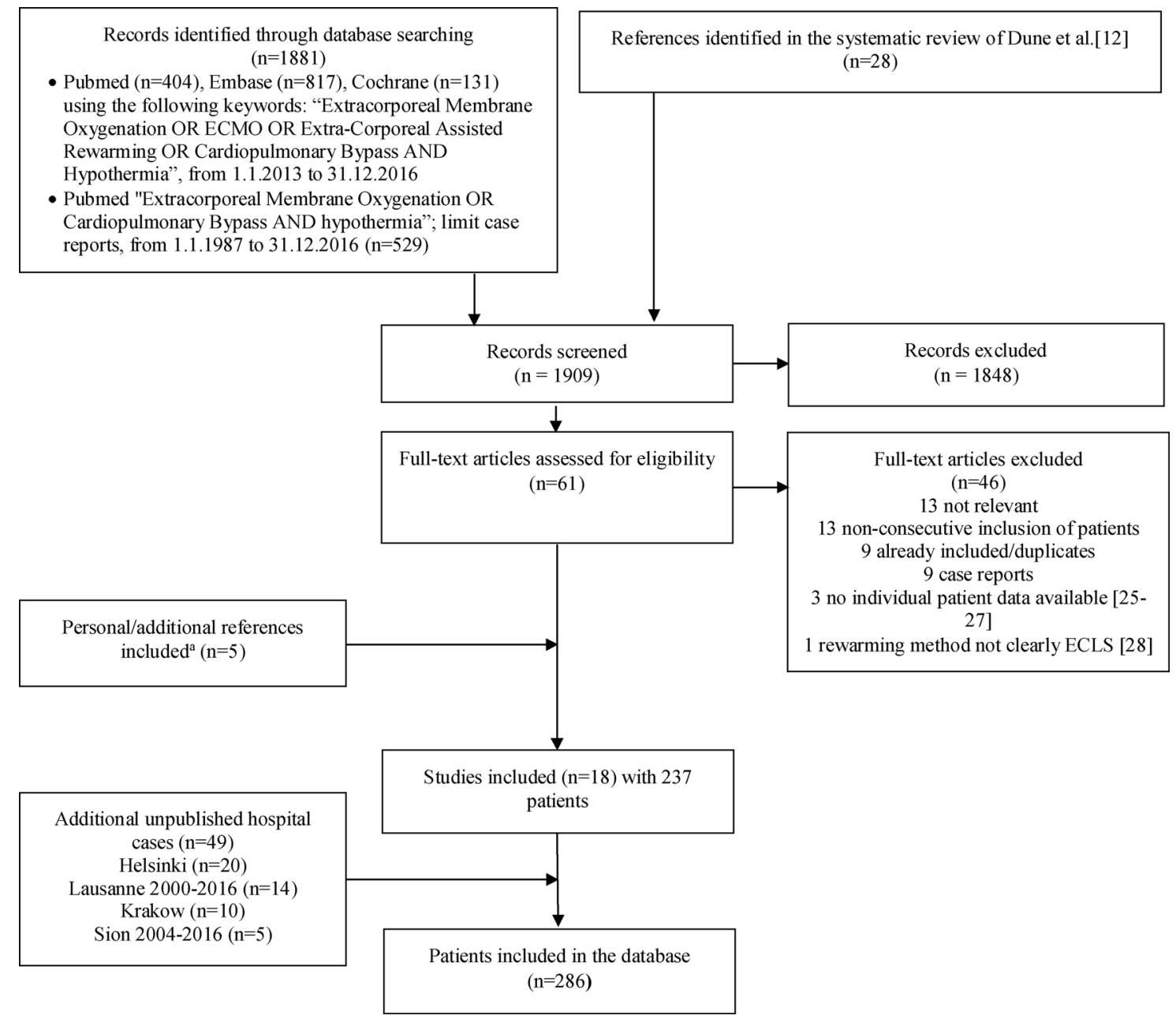

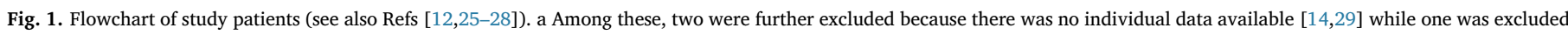
because the rewarming method was not clearly Extracorporeal Life Support [30]. ECLS = Extracorporeal Life Support.

\section{Results}

The literature search generated 1881 publications. Eighteen studies were included, for a total of 237 patients. The hospital data collection resulted in 49 additional patients (Fig. 1, eTables 1 and 2). Out of the 286 included patients, 106 survived and 180 died, resulting in an overall survival of $37 \%$ (95\% CI: 32-43\%), with a CPC of 1 or 2 for 84 $(84 \%)$ of the 100 survivors for whom this information was available.

Univariate associations between the nine predictors and survival are summarized for both the categorical (Table 1) and the continuous predictors (Table 1, eFig. 1). The backward model selection procedure (eTable 3 and eStatistics) resulted in a final model including six predictors and eight parameters yielding the following score: score $\mathbf{= 2 . 4 4}$

- $1.55 \times$ male $-1.95 \times$ (asphyxia -related mechanism) $-0.0191 \times$ age $-2.07 \times \log _{2}$ potassium $-0.573 \times \log _{2} \quad$ (CPR

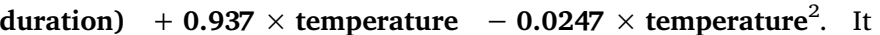
should be noted that this score is expressed on the logit scale. The Hypothermia Outcome Prediction after ECLS (HOPE) survival probability score can then be simply obtained from the score by calculating: HOPE survival probability $=\exp ($ score $) /(1+\exp ($ score $))$. An example of a HOPE survival probability score is provided as a supplementary file (eExample). A dedicated calculator of the HOPE survival probability score, together with a $95 \%$ confidence interval, is available online at www.hypothermiascore.org.

The boxplots of HOPE survival probabilities estimated for our 286 patients are presented in Fig. 2. The area under the corresponding receiver operating characteristic curve (AUC) was 0.895 (95\% CI:
0.859-0.931) compared to 0.774 (95\% CI: 0.720-0.828) when based on serum potassium level alone. Using our internal validation procedure (Additional file 2: Statistics), we estimated an excess of optimism of 0.029 , such that our corrected estimation of AUC would be of 0.866 . Of note, the year of data collection $(p=0.665)$, and whether data had been published $(\mathrm{p}=0.514)$, were not statistically significant when added into our final analysis. Further, introducing the hospital as an additional factor in our final model did not improve the AIC (AIC $=240.8$ for our final model when averaged over the 100 imputed data sets), whether treated as a fixed effect (AIC $=246.0$ ) or as a random effect (AIC $=242.3$ )

The sensitivities and specificities based on the traditional triage criteria and those estimated from our data are provided in Table 2. While the sensitivity of temperature or potassium criteria was high (99-100\% for any criterion), the specificity was at most $23 \%$ (for potassium $>8 \mathrm{mmol} / \mathrm{L}$ ) and was only $8 \%$ (95\% CI: 5-13\%) for the commonly applied criterion potassium $>12 \mathrm{mmol} / \mathrm{L}$. These results were improved using the HOPE survival probability score, the specificity reaching 51\% (95\% CI: 45-60\%) if using criterion HOPE $<0.1$ as a criterion for a poor prognosis (Table 2, Fig. 2). Calculated over the entire sample of patients and using the same cutoff of 0.1 for the HOPE criterion, the proportion of patients estimated to survive but who died (false positives) was 31\% (95\% CI: $25-36 \%$ ) compared to $58 \%$ (95\% CI: $52-63 \%$ ) using potassium $\leq 12 \mathrm{mmol} / \mathrm{L}$ as a rewarming criterion (Fig. 3). The proportion of patients estimated to die but who survived (false negatives) was estimated to be $0 \%$ (95\% CI: $0-1.7 \%$ for HOPE, $0-1.4 \%$ for potassium) in both situations. 
Table 1

Univariate associations between potential predictors and survival. All predictors apart the rewarming method were significantly associated with survival.

\begin{tabular}{|c|c|c|c|c|}
\hline & Overall $(\mathrm{n}=286)$ & Survivors $106 / 286=37 \%$ & Non-survivors $180 / 286=63 \%$ & $\mathrm{P}$ value \\
\hline \multicolumn{5}{|c|}{ Continuous potential predictors, median (IQR) } \\
\hline Age (years) & $35(16-55)$ & $40(18-56)$ & $29.5(13-54)$ & 0.035 \\
\hline Temperature $\left({ }^{\circ} \mathrm{C}\right)$ & $24(22-27)$ & $23(21-25)$ & $25(22-28)$ & $<0.001$ \\
\hline Potassium (mmol/L) & $4.7(3.6-6.6)$ & $3.8(3.1-4.65)$ & $5.8(4.0-8.0)$ & $<0.001$ \\
\hline CPR duration (min) & $120(85-169)$ & $106(64-165)$ & $120(90-169)$ & 0.013 \\
\hline \multicolumn{5}{|c|}{ Categorical potential predictors, $\mathrm{n}(\%)$} \\
\hline Gender & & & & $\mathrm{p}<0.001$ \\
\hline Female & $71 / 286=25 \%$ & $43 / 71=61 \%$ & $28 / 71=39 \%$ & \\
\hline Male & $215 / 286=75 \%$ & $63 / 215=29 \%$ & $152 / 215=71 \%$ & \\
\hline Mechanism & & & & $\mathrm{p}<0.001$ \\
\hline Exposure & $98 / 283=35 \%$ & $56 / 98=57 \%$ & $42 / 98=43 \%$ & \\
\hline Immersion & $40 / 283=14 \%$ & $24 / 40=60 \%$ & $16 / 40=40 \%$ & \\
\hline Submersion & $94 / 283=33 \%$ & $18 / 94=19 \%$ & $76 / 94=81 \%$ & \\
\hline Avalanche & $51 / 283=18 \%$ & $6 / 51=12 \%$ & $45 / 51=88 \%$ & \\
\hline Cardiac rhythm & & & & $\mathrm{p}<0.001$ \\
\hline Asystole & $139 / 256=54 \%$ & $32 / 139=23 \%$ & $107 / 139=77 \%$ & \\
\hline Ventricular fibrillation & $91 / 256=36 \%$ & $49 / 91=54 \%$ & $42 / 91=46 \%$ & \\
\hline PEA & $26 / 256=10 \%$ & $18 / 26=69 \%$ & $8 / 26=31 \%$ & \\
\hline CA Circumstance & & & & $\mathrm{p}<0.001$ \\
\hline Unwitnessed CA & $165 / 250=66 \%$ & $44 / 165=27 \%$ & $121 / 165=73 \%$ & \\
\hline Witnessed CA & $85 / 250=34 \%$ & $48 / 85=56 \%$ & $37 / 85=44 \%$ & \\
\hline Type of ECLS & & & & $\mathrm{P}=0.228$ \\
\hline CPB & $201 / 286=70 \%$ & $70 / 201=35 \%$ & $131 / 201=65 \%$ & \\
\hline ECMO & $85 / 286=30 \%$ & $36 / 85=42 \%$ & $49 / 85=58 \%$ & \\
\hline
\end{tabular}

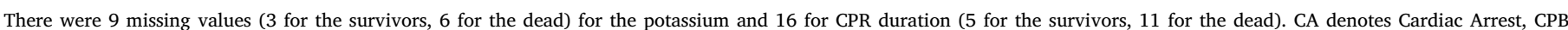
Cardiopulmonary Bypass, CPR Cardiopulmonary Resuscitation, ECLS Extracorporeal Life Support ECMO Extracorporeal Membrane Oxygenation, PEA Pulseless Electrical Activity.

\section{Discussion}

As far as we are aware, our study is the largest to date that assesses outcome of hypothermic arrested patients with ECLS rewarming and the first to predict survival with ECLS in arrested patients using a multivariable model. Based on our results, the prediction of survival probability to hospital discharge was both statistically and clinically significantly improved if estimated with our score instead of a traditional dichotomous potassium-based triage decision. Our mixed method of retrospective data collection from different sources circumvented the major limitation of all studies reporting survival after hypothermic CA, namely their small sample size, ranging from case reports to case series with patient numbers in low double digits $[3,13,31,32]$. The prospective inclusion of sufficient patients for a study of this nature would take years, even if several international centers cooperated to conduct a multivariable analysis comparable to our study. With nearly 300 patients from different centers worldwide, which is to our knowledge the largest sample size by far for arrested hypothermic patients, our study allowed us to test for predictors potentially associated with survival, using an approach designed to minimize the risk of inclusion bias by including consecutive patients.

There are several key features to this study. First, we confirm the association of six independent predictors of survival that had proven controversial in previous studies: sex; asphyxia-related mechanism of CA; age; potassium level; CPR duration; and temperature $[9,14,27,29,33,34]$. On the other hand, the significant univariate association between CA (witnessed vs. unwitnessed), described in several recent studies $[13,35,36]$, was not retained as a predictive variable once
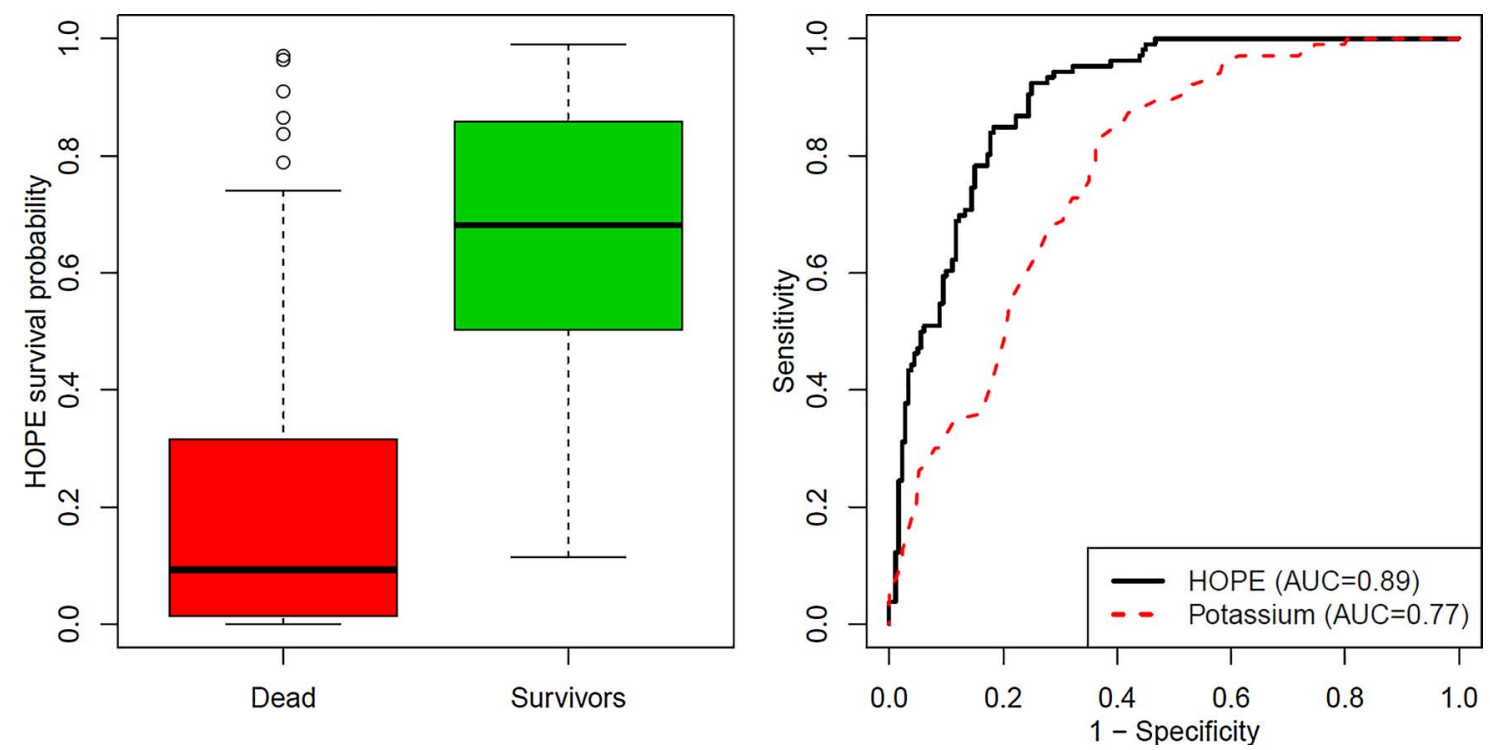

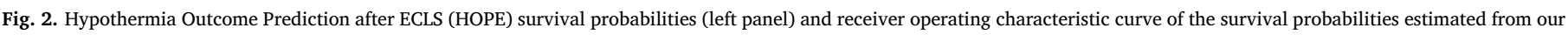
286 patients using Hypothermia Outcome Prediction after ECLS (HOPE) model or potassium (right panel). AUC = area under the curve. 
Table 2

Estimated sensitivity and specificity based on different criteria. HOPE denotes Hypothermia Outcome Prediction after ECLS.

\begin{tabular}{|c|c|c|c|c|c|c|}
\hline Criterion (good outcome) ${ }^{a}$ & Sensitivity $^{\mathrm{b}}$ & Specificity $^{\mathrm{c}}$ & $\mathrm{PPV}^{\mathrm{d}}$ & $\mathrm{NPV}^{\mathrm{e}}$ & $\mathrm{FP}^{\mathrm{f}}$ & $\mathrm{FN}^{\mathrm{f}}$ \\
\hline Potassium $\leq 12 \mathrm{mmol} / \mathrm{L}$ & $103 / 103=100 \%$ & $14 / 174=8 \%$ & 39 & 100 & 58 & 0.0 \\
\hline Potassium $\leq 8 \mathrm{mmol} / \mathrm{L}$ & $102 / 103=99 \%$ & $40 / 174=23 \%$ & 43 & 98 & 48 & 0.4 \\
\hline HOPE $\geq 0.05$ & $106 / 106=100 \%$ & $69 / 180=38 \%$ & 49 & 100 & 39 & 0.0 \\
\hline HOPE $\geq 0.10$ & $106 / 106=100 \%$ & $92 / 180=51 \%$ & 55 & 100 & 31 & 0.0 \\
\hline $\mathrm{HOPE} \geq 0.20$ & $101 / 106=95 \%$ & $115 / 180=64 \%$ & 61 & 96 & 23 & 1.7 \\
\hline $\mathrm{HOPE} \geq 0.30$ & $98 / 106=92 \%$ & $130 / 180=72 \%$ & 66 & 94 & 17 & 2.8 \\
\hline $\mathrm{HOPE} \geq 0.40$ & $90 / 106=85 \%$ & $147 / 180=82 \%$ & 73 & 90 & 12 & 5.6 \\
\hline HOPE $\geq 0.50$ & $81 / 106=76 \%$ & $153 / 180=85 \%$ & 75 & 86 & 9 & 8.7 \\
\hline
\end{tabular}

${ }^{\text {a }}$ Nine values were missing for potassium.

b The sensitivity is defined as the probability that the criterion is fullfilled among the survivors.

${ }^{c}$ The specificity is defined as the probability that the criterion is not fullfilled among the non-survivors.

${ }^{d}$ The Positive Predictive Value is defined as the proportion of patients who survive among those fulfilling the criterion.

e The Negative Predictive Value is defined as the proportion of patients who dies among those not fulfilling the criterion.

${ }^{f}$ FP denote the percentage of false positive and FN the percentage of false negative (calculated over all the patients, whether positive or negative).

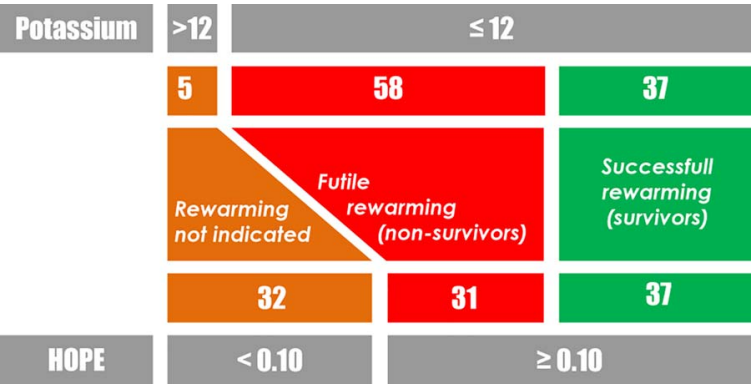

Fig. 3. Comparison of the outcome and proportion of patients for whom rewarming is indicated according to the HOPE vs potassium triage. In a situation where a physician would rewarm only those patients achieving a good prognosis, when facing 100 patients, 27 ECLS unsuccessful rewarming could be avoided without losing a single additional life using our score (with a cutoff at 0.10 ) rather than potassium alone (with a cutoff of $12 \mathrm{mmol} / \mathrm{L}$ ) as a rewarming criterion.

entered in our multivariable analysis.

A second key feature is that our results support a paradigm shift from a dichotomous potassium triage $[1,6,7]$ to a more comprehensive decision-making tool for patients arrested due to accidental hypothermia. Hyperkaliemia has long been known to be associated with poor prognosis in severe hypothermia [5]. The dichotomous potassium approach was proposed in 1990, when a key study found that serum potassium levels on admission in non-survivors were extremely high (14.5 mmol/L; range, $6.8-24.5 \mathrm{mmol} / \mathrm{L})$ compared to survivors (3.5 mmol/L; range, $2.7-5.3 \mathrm{mmol} / \mathrm{L})$ [4]. The same year, another group of investigators proposed ECLS rewarming as a promising therapeutic tool to treat hypothermic arrested patients. Five of eleven patients who had received CPB rewarming survived with good neurological outcome [37].

Subsequently, attempts have been made to refine the potassium triage in accidentally hypothermic CA patients, including hypothermic avalanche patients in CA [38]. Instead of using a specific cutoff, our model uses the prognostic information provided by particular potassium levels. In one scenario (Additional file 3: Example) a potassium of $3.5 \mathrm{mmol} / \mathrm{L}$ is associated with a probability of survival of nearly $70 \%$. This decreases to $59 \%$ with a potassium of $4 \mathrm{mmol} / \mathrm{L}$ and to $30 \%$ with a potassium of $6 \mathrm{mmol} / \mathrm{L}$, all other parameters remaining equal. Thus, our score is in accordance with studies where normal or even low serum potassium levels are observed at the onset of hypothermia [39]. A normal or low potassium level may be a marker of a short duration of CA indicative of better prognosis, even if it is still associated with a $30 \%$ risk of death in our example. Also, hypothermic patients may present with hypokalemia owing to several pathophysiological mechanisms, such as temperature-induced reduction of physiological processes, intracellular shift of potassium due to change in $\mathrm{pH}$ or $\beta 2$-adrenergic receptor stimulation, or finally a hypothermia-membrane-stabilizing effect $[40,41]$. If serum potassium level is a parameter influenced by several factors beside heart or brain cell death, it seems unwise to base a life-or-death decision on this parameter alone.

The third and most important key feature is that estimates of survival probability using our new score are more accurate than those based on the present serum potassium-based triage and may provide better guidance for decision-making. An accurate prediction of survival is important to avoid both over- or undertreatment. Based on anecdotal reports and expert opinions, clinicians who are not familiar with hypothermic arrest may not start ECLS rewarming as they may underestimate the true patient's survival probability. Their judgment may be miscalibrated if based on the dismal survival of out-of-hospital cardiac arrest of normothermic patients.

Avoiding undertreatment is especially important, because the neurological outcome of survivors are significantly better than the neurologic outcomes of patients who survived ECLS after normothermic cardiac arrest [17]. On the other side, use of our score instead of the potassium threshold over the entire sample would have reclassified $27 \%$ of patients, avoiding one in four futile ECLS rewarming attempts, without losing a single additional life. Our model helps to direct limited resources of financially strained health care systems to those patients with a better chance to benefit, especially when a reliable triage of multiple patients is necessary.

Finally, this study provides a practical software tool, which enables an estimate of the survival chances of a person in hypothermic CA. This tool can be easily accessed via the internet as a web application, and allows a rapid assessment of survival chances. A more accurate estimate of prognosis may also improve informed discussion with relatives pertaining to the initiation and outcome of ECLS rewarming.

\section{Limitations}

Our study suffers from some limitations. The first is the potential of selection bias owing to the retrospective nature of the study. We were unable to analyze patients for whom the clinicians decided against ECLS rewarming for whatever reason, nor patients declared dead at the scene. This selection bias may have led to an overestimation of the survival rate. Also, not having all the deaths in our sample may have induced some bias in some of our estimates such as specificities, which would actually be underestimated if those deaths would have achieved a low HOPE score. However, the retrospective design was necessary because of the relative rarity of hypothermic patients in CA. We tried to minimize the risk of inclusion bias by including only consecutive patients. Another potential limitation is the heterogeneity of caseload and experience of the centers we included. However, the lack of statistical evidence for a "center effect" in this study suggests potential good external validity, although independent validation is still necessary. 
Because of the difficulties linked to the prospective collection of sufficient amount of data, external validation would be best performed by using existing consecutive cases not included in this derivation cohort. The fact that the HOPE score cannot be calculated with missing values may also be considered as a limitation. Finally, our literature search was based on a previously published systematic literature review [12] whose quality and methodology was assumed to be good.

\section{Conclusions}

In this large retrospective study we found that our multivariable model was superior to dichotomous triage based on serum potassium level in predicting which hypothermic patients in cardiac arrest would benefit from extracorporeal life support. This might be useful to improve the decision-making process when considering the rewarming of a hypothermic arrested patient. External validation of our findings is required.

\section{Funding source}

None.

\section{Conflict of interest}

None to declare. We, all of the authors, have no financial or personal relationships with other people or organisations that could have inappropriately influenced our work.

\section{Acknowledgements}

For their contribution to the acquisition of the data: Prof. Pierre Bouzat, France; Dr Guillaume Debaty, France; Prof. Christoph Bernhard Eich, Germany; Dr Sylweriusz Kosiński, Poland; Prof. Dr Katsunori Mochizuki, Japan; Prof. Jacob Eifer Møller, Danmark; Dr Seiji Morita, Japan; Dr Matthieu de Riedmatten, Switzerland. For her help in the literature search: Mme Cécile Jaques, Bibliothèque Universitaire de Médecine de Lausanne, Switzerland. For the final proofreading and translation: Dr Michael Cotton. For the creation of the internet website: Dr Alexandre Gnaegi, Switzerland. For the revision of the internet website: Dr Fabrice Dami, Switzerland. For his unconditional support: Prof. Bertrand Yersin, Switzerland.

\section{Appendix A. Supplementary data}

Supplementary data associated with this article can be found, in the online version, at https://doi.org/10.1016/j.resuscitation.2018.02.026.

\section{References}

[1] Brown DJ, Brugger H, Boyd J, Paal P. Accidental hypothermia. N Engl J Med 2012;367:1930-8.

[2] Meiman J, Anderson H, Tomasallo C. Hypothermia-related deaths - Wisconsin, 2014, and United States, 2003-2013. Centers Dise Control Prev Morbidity Mortality Wkly Rep (MMWR) 2015;64:141-3.

[3] Walpoth BH, Walpoth-Aslan BN, Mattle HP, Radanov BP, Schroth G, Schaeffler L, et al. Outcome of survivors of accidental deep hypothermia and circulatory arrest treated with extracorporeal blood warming. N Engl J Med 1997;337:1500-5.

[4] Schaller MD, Fischer AP, Perret CH. Hyperkalemia. A prognostic factor during acute severe hypothermia. JAMA 1990;264:1842-5.

[5] Hauty MG, Esrig BC, Hill JG, Long WB. Prognostic factors in severe accidental hypothermia: experience from the Mt. Hood tragedy. J Trauma 1987;27:1107-12.

[6] Paal P, Gordon L, Strapazzon G, Brodmann Maeder M, Putzer G, Walpoth B, et al. Accidental hypothermia-an update: the content of this review is endorsed by the International Commission for Mountain Emergency Medicine (ICAR MEDCOM). Scand J Trauma Resusc Emerg Med 2016;24:111.

[7] Truhlář A, Deakin CD, Soar J, Khalifa GE, Alfonzo A, Bierens JJ, et al. Cardiac arrest in special circumstances section collaborators: European resuscitation council guidelines for resuscitation 2015: section 4. Cardiac arrest in special circumstances. Resuscitation 2015;95:148-201.

[8] Vanden Hoek TL, Morrison LJ, Shuster M, Donnino M, Sinz E, Lavonas EJ, et al. Part 12: cardiac arrest in special situations: 2010 American Heart Association guidelines for cardiopulmonary resuscitation and emergency cardiovascular care. Circulation 2010;122:S829-61.

[9] Farstad M, Andersen KS, Koller ME, Grong K, Segadal L, Husby P. Rewarming from accidental hypothermia by extracorporeal circulation: a retrospective study. J Cardiothorac Surg 2001;20:58-64.

[10] Van Tilburg C, Grissom CK, Zafren K, McIntosh S, Radwin MI, Paal P, et al. Wilderness medical society practice guidelines for prevention and management of avalanche and nonavalanche snow burial accidents. Wilderness Environ Med 2017;28:23-42.

[11] Martinell L, Nielsen N, Herlitz J, Karlsson T, Horn J, Wise MP, et al. Early predictors of poor outcome after out-of-hospital cardiac arrest. Crit Care 2017;21:96.

[12] Dunne B, Christou E, Duff O, Merry C. Extracorporeal-assisted rewarming in the management of accidental deep hypothermic cardiac arrest: a systematic review of the literature. Heart Lung Circ 2014;23:1029-35.

[13] Debaty G, Moustapha I, Bouzat P, Maignan M, Blancher M, Rallo A, et al. Outcome after severe accidental hypothermia in the French Alps: a 10-year review. Resuscitation 2015;93:118-23.

[14] Ruttmann E, Weissenbacher A, Ulmer H, Müller L, Höfer D, Kilo J, et al. Prolonged extracorporeal membrane oxygenation-assisted support provides improved survival in hypothermic patients with cardiocirculatory arrest. J Thorac Cardiovasc Surg 2007; 134:594-600.

[15] Gilbert EH, Lowenstein SR, Koziol-McLain J, Barta DC, Steiner J. Chart reviews in emergency medicine research: where are the methods. Ann Emerg Med $1996 ; 27: 305-8$

[16] Cummins RO, Chamberlain DA, Abramson NS, Allen M, Baskett PJ, Becker L, et al. Recommended guidelines for uniform reporting of data from out-of-hospital cardiac arrest: the Utstein Style. A statement for health professionals from a task force of the American Heart Association, the European Resuscitation Council, the Heart and Stroke Foundation of Canada, and the Australian Resuscitation Council. Circulation 1991;84:960-75.

[17] Ouweneel DM, Schotborgh JV, Limpens J, Sjauw KD, Engström AE, Lagrand WK, et al. Extracorporeal life support during cardiac arrest and cardiogenic shock: a systematic review and meta-analysis. Intensive Care Med 2016;42:1922-34.

[18] Safar P. Resuscitation after brain ischemia. In: GAaSPeditor. Brain failure and resuscitation. New York: Churchill Livingstone; 1981. p. 155-84.

[19] Burnham P, Anderson DR. Model Selection and Multimodel Inference a Practical Information-theoretic Approach. New York: Springer-Verlag; 2002.

[20] Ambler G, Brady AR, Royston P. Simplifying a prognostic model: a simulation study based on clinical data. Stat Med 2002;21:3803-22.

[21] van Buuren S, Groothuis-Oudshoorn K. MICE. Multivariate imputation by chained equations in R. J Stat Softw 2011;45:1-67.

[22] R Core Team. Computing RFfSeditor. A language and environment for statistical computing. 2016.

[23] Rubin DB. Multiple imputation for nonresponse in surveys. New York: John Wiley \& Sons; 1987.

[24] Steyerberg EW, Harrell FEJ, Borsboom GJ, Eijkemans MJ, Vergouwe Y, Habbema JD. Internal validation of predictive models: efficiency of some procedures for logistic regression analysis. J Clin Epidemiol 2001;54:774-81.

[25] Sawamoto K, Bird SB, Katayama Y, Maekawa K, Uemura S, Tanno K, et al. Outcome from severe accidental hypothermia with cardiac arrest resuscitated with extracorporeal cardiopulmonary resuscitation. Am J Emerg Med 2014;32:320-4.

[26] Coskun KO, Popov AF, Schmitto JD, Hinz J, Kriebel T, Schoendube FA, et al. Extracorporeal circulation for rewarming in drowning and near-drowning pediatric patients. Artif Organs 2010;34:1026-30.

[27] Hilmo J, Naesheim T, Gilbert M. Nobody is dead until warm and dead: prolonged resuscitation is warranted in arrested hypothermic victims also in remote areas-a retrospective study from northern Norway. Resuscitation 2014;85:1204-11.

[28] Brunette DD, McVaney K. Hypothermic cardiac arrest: an 11 year review of ED management and outcome. Am J Emerg Med 2000;18:418-22.

[29] Mair P, Brugger H, Mair B, Moroder L, Ruttmann E. Is extracorporeal rewarming indicated in avalanche victims with unwitnessed hypothermic cardiorespiratory arrest. High Alt Med Biol 2014:15:500-3.

[30] Currie PT, Brunette DD. Hypothermic cardiac arrest: an update. Am J Emerg Med 2006;24:389-90.

[31] Khorsandi M, Dougherty S, Young N, Kerslake D, Giordano V, Lendrum R, et al. Extracorporeal life support for refractory cardiac arrest from accidental hypothermia: a 10-year experience in edinburgh. J Emerg Med 2017;52:160-8.

[32] Schober A, Sterz F, Handler C, Kürkciyan I, Laggner A, Röggla M, et al. Cardiac arrest due to accidental hypothermia-a 20 year review of a rare condition in an urban area. Resuscitation 2014;85:749-56.

[33] Debaty G, Babaz V, Durand M, Gaide-Chevronnay L, Fournel E, et al. Prognostic factors for extracorporeal cardiopulmonary resuscitation recipients following outof-hospital refractory cardiac arrest. A systematic review and meta-analysis. Resuscitation 2017;112:1-10.

[34] Svendsen OS, Grong K, Andersen KS, Husby P. Outcome after rewarming from accidental hypothermia by use of extracorporeal circulation. Ann Thorac Surg 2017;103:920-5.

[35] Moroder L, Mair B, Brugger H, Voelckel W, Mair P. Outcome of avalanche victims with out-of-hospital cardiac arrest. Resuscitation 2015;89:114-8.

[36] Boué Y, Payen JF, Brun J, Thomas S, Levrat A, Blancher M, et al. Survival after avalanche-induced cardiac arrest. Resuscitation 2014;85:1192-6.

[37] Walpoth BH, Locher T, Leupi F, Schüpbach P, Mühlemann W, Althaus U. Accidental deep hypothermia with cardiopulmonary arrest: extracorporeal blood rewarming in 11 patients. Eur J Cardiothorac Surg 1990;4:390-3.

[38] Cohen JG, Boué Y, Boussat B, Reymond E, Grand S, Blancher M, et al. Serum potassium concentration predicts brain hypoxia on CT after avalanche-induced 
cardiac arrest. Am J Emerg Med 2016;34:856-60.

[39] Strapazzon G, Falk M, Paal P, Brugger H. The challenge of establishing a correct serum potassium cutoff for inhospital triage after avalanche-induced cardiac arrest. Am J Emerg Med 2016;34:1317.

[40] Buse S, Blancher M, Viglino D, Pasquier M, Maignan M, Bouzat P, et al. The impact of hypothermia on serum potassium concentration: a systematic review. Resuscitation 2017:118:35-42.

[41] Aibiki M, Kawaguchi S, Maekawa N. Reversible hypophosphatemia during moderate hypothermia therapy for brain-injured patients. Crit Care Med 2001;29:1726-30. 